\title{
Notas Botánicas
}

\section{Identificación de la madera de varias esculturas de origen olmeca}

En febrero de 1988, los lugareños de los poblados de El Manatí y El Macayal, del municipio de Hidalgotitlán, Veracruz, al hacer excavaciones para construir pozas piscícolas, encontraron material arqueológico consistente en un conjunto de objetos como hachas de piedra, restos humanos y otras piezas entre las cuales llamaron la atención de arqueólogos y antropólogos, 13 esculturas de madera, consistentes en bustos antropomorfos de aproximadamente $49 \mathrm{~cm}$ de altura cada uno.

El grupo de investigadores del Instituto de Investigaciones Antropológicas, UNAM y del Instituto Nacional de Antropología e Historia, encargados del proyecto "Manatî", nos solicitó (por medio del Dr. Paul Schmidt) la identificación de la madera de dichas esculturas.

En su informe preliminar sobre El Manatí, Ortiz, et al. (1988) anotan que las esculturas poseen una antiguedad de $2990 \pm 150$ años ya que datan aproximadamente de 1183 a 883 años a.c. y que su característica más sobresaliente es la ausencia intencional de brazos. También mencionan que sus rasgos son típicos de la escultura olmeca: las orejeras, la cabeza rapada, la nariz afilada y los ojos oblicuos (fig. 1).

Es interesante hacer notar que las trece esculturas muestran una amplia variedad en sus rasgos faciales y forma de las caras, por lo que aparentemente representan personajes diferentes, e incluso existe un parecido en algunas de estas esculturas con otras figuras de la cultura olmeca encontradas con anterioridad y esculpidas en piedra, como "El Luchador" o el "Señor de las Limas".

La región donde fueron encontradas las esculturas se conoce como el cerro El Manatí el cual se localiza en la cuenca baja del río Coatzacoalcos; es una área rodeada de pantanos a $2.5 \mathrm{~km}$ del poblado El Macayal, aproximadamente a los $94^{\circ} 37^{\prime} \mathrm{W}-17^{\circ} 40^{\prime} \mathrm{N}$ (fig. 2). El clima, de acuerdo con la estación meteorológica más cercana (Hidalgotitlán, Ver.), es del tipo Amw"(i)g, cálido húmedo con lluvias de verano, con temperatura media anual de $25.7^{\circ} \mathrm{C}$ y $2,014 \mathrm{~mm}$ de precipitación anual (García, 1981).

Geológicamente el cerro Manatí es un domo salino del jurásico que además contiene azufre (Schmidt com. pers.).

En cuanto a la metodología utilizada para llegar a la identificación de la madera en que están talladas las figuras, de cada una se tomó una muestra pequeña (aprox. $1 \mathrm{~cm}$ ) que se colocó inmediatamente en FAA, y después se lavó durante 48 hrs. Posteriormente

Barajas-Morales J. 1991. Identificación de la madera de varias esculturas de origen olmeca. Boletín de la Sociedad Botánica de México 51: 83-92. 
BOLETIN DE LA SOCIEDAD BOTANICA DE MEXICO, Núm. 51, 1991
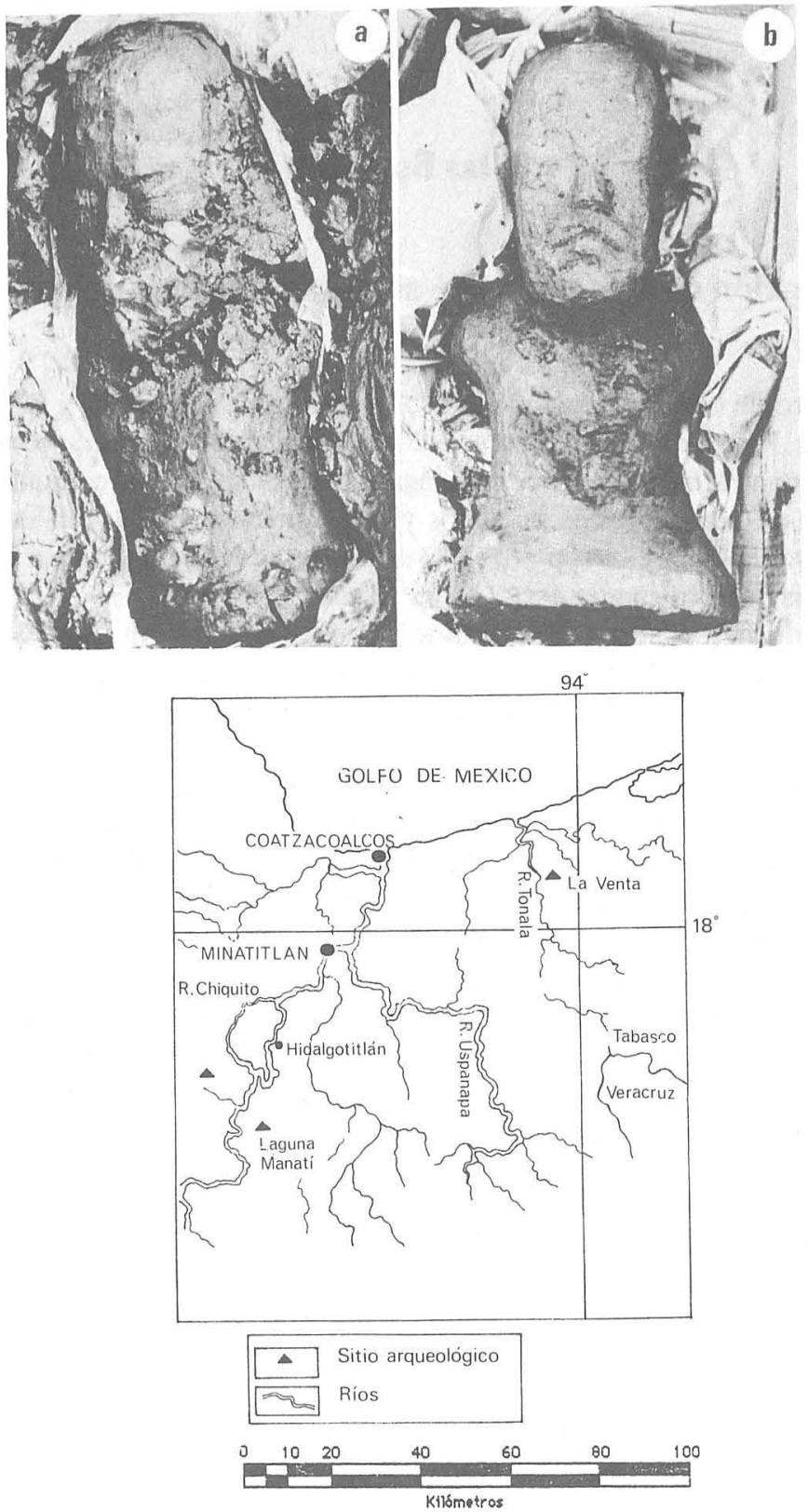

Fig. 1. Aspecto de las alturas 12 y 13 encontradas en el sitio "El Manati" (Foto Paul Schmidt). Fig. 2. Mapa de localización del sitio "El Manatî", en el estado Veracruz. 
todas las muestras fueron deshidratadas con una mezcla de alcohol butílico y etanol e incluidas en parafina, de acuerdo con la secuencia y proporciones indicadas por Sass - (1958). Una vez incluidas todas las muestras, cada bloque se cortó en un microtomo rotatorio para obtener cortes transversales, tangenciales y radiales de $20 \mu$ de grosor aproximado, que posteriormente fueron teñidos con verde rápido y montados en resina sintética.

Los resultados muestran que 9 de las esculturas fueron fabricadas con madera de Spondias mombin L. y 4 con la de Ceiba pentandra (L.) Gaertn. (tabla 1).

Las características que pudieron ser observadas en las muestras y que facilitaron su identificación, se dan a continuación:

\section{Spondias mombin L. Familia ANACARDIACEAE. \\ Nombre común: jobo \\ Muestras Núms. 1,3,4,5,6,10,11,12 y 13 (fig. 3)}

Madera con porosidad difusa con poros ovalados, principalmente solitarios y algunos en grupos de 2 , moderadamente pocos, $8 / \mathrm{mm}^{2}$ y medianos con diámetro tangencial de 203 (169-301) $\mu$. Elementos de vaso con platina de perforación simple, punteaduras intervasculares alternas, las de vaso a radio grandes y alargadas y presencia de tílides escasas.

TABLA 1. Tamaño de las esculturas y especie

\begin{tabular}{ccccc}
\hline Núm. & $\begin{array}{c}\text { altura } \\
\text { cm }\end{array}$ & $\begin{array}{c}\text { ancho } \\
\mathrm{cm}\end{array}$ & $\begin{array}{c}\text { espesor } \\
\mathrm{cm}\end{array}$ & especie \\
1 & 45.5 & 26 & 10 & S. mombin \\
2 & 33 & 13 & 13 & C. pentandra \\
3 & -- & -- & -- & S. mombin \\
4 & 45 & 22 & 11 & S. mombin \\
5 & 41.5 & 19 & 14 & S. mombin \\
6 & 43 & 19 & 14 & S. mombin \\
7 & 42 & 20 & 18 & C. pentandra \\
8 & 36 & 13 & 11 & C. pentandra \\
9 & 23 & 12 & 10 & S. montandra \\
10 & -- & -- & -- & S. mombin \\
11 & 49.5 & 27 & 16 & S. mombin \\
12 & 43 & 8 & -- & S. mombin \\
13 & 48 & -- & -- & \\
\hline
\end{tabular}




\section{células.}

El parénquima axial es paratraqueal vasicéntrico con series parenquimáticas de 4

Los radios son heterocelulares tipos II y III (Kribs 1968), multiseriados de 4 a 8 células; con células procumbentes en el cuerpo y cuadradas y erectas en los márgenes. Son moderadamente numerosos, $5 / \mathrm{mm}$ y muy bajos, con altura de $756 \mu$ en promedio, algunos presentan gomas escasas.

Las fibras son libriformes y septadas, con 1 ó 2 septos. Se presentan algunos canales intercelulares radiales.

\section{Ceiba pentandra (L.) Gaertn. Familia BOMBACACEAE. Nombre común: pochote o ceiba. Muestras Núms. 2,7,8 y 9 (fig. 4)}

Madera con porosidad difusa, poros circulares principalmente solitarios, escasos, $4 / \mathrm{mm}^{2}$ y algo grandes, con diámetro tangenciall de $207(160-236) \mu$. Platina de perforación simple; punteaduras intervasculares alternas y de vaso a radio más grandes y alargadas.

El parénquima axial es apotraqueal en bandas tangenciales uniseriadas abundante con series parenquimáticas de 4 células.

Los radios son heterocelulares tipo III (Kribs, 1968) multiseriados de 5 a 9 células; con células cuadradas en el cuerpo y erectas en los márgenes; moderadamente numerosos, $4 / \mathrm{mm}$ y moderadamente bajos, con altura promedio de $2350 \mu$. Presentan células envolventes y están parcialmente estratificados (3 líneas $/ \mathrm{mm}$ ). Las fibras son libriformes muy escasas.

En las figuras 3 y 4 se observan las características de los especímenes arqueológicos identificados, y pueden compararse con las muestras actuales de las mismas especies que se presentan en las figuras 5 y 6 respectivamente.

En la actualidad aun se encuentran en la región, abundantes árboles de Spondias mombin y Ceiba pentandra, ambas especies poseen maderas ligeras y blandas, con una gravedad específica de 0.43 y 0.40 respectivamente (Kribs, 1968), ésto las hace un material ideal para labrar y trabajar, pero al mismo tiempo son tremendamente suceptibles al daño por insectos, hongos o cualquier otro elemento que deteriore la madera; de aquí que llame tremendamente la atención la conservación de estas esculturas fabricadas con especies tan suceptibles al daño.

La dureza de la madera de las esculturas se observó notablemente disminuida encontrándose incluso comprimida en algunas áreas, no obstante la estructura se conservó relativamente bien gracias a lo cual se logró la identificación de este material.

Es evidente que el conocimiento certero de las especies con las que fueron construidas dichas esculturas, será de enorme trascendencia para el conocimiento de la cultura Olmeca, ya que por una parte contribuirá a comprobar algunos datos ya conocidos, por ejemplo, el hecho de que el árbol de ceiba era considerado como un 
elemento sagrado utilizado especialmente para ceremonias religiosas; por otra parte, una conclusión de mayor importancia es la confirmación de que esta cultura poseía un gran conocimiento de sus especies vegetales y de las características y propiedades que cada una tenía pues en este caso es claro que por tratarse de especies blandas y por lo tanto fáciles de trabajar, resultan muy adecuadas para la fabricación de esculturas y otros implementos labrados. 


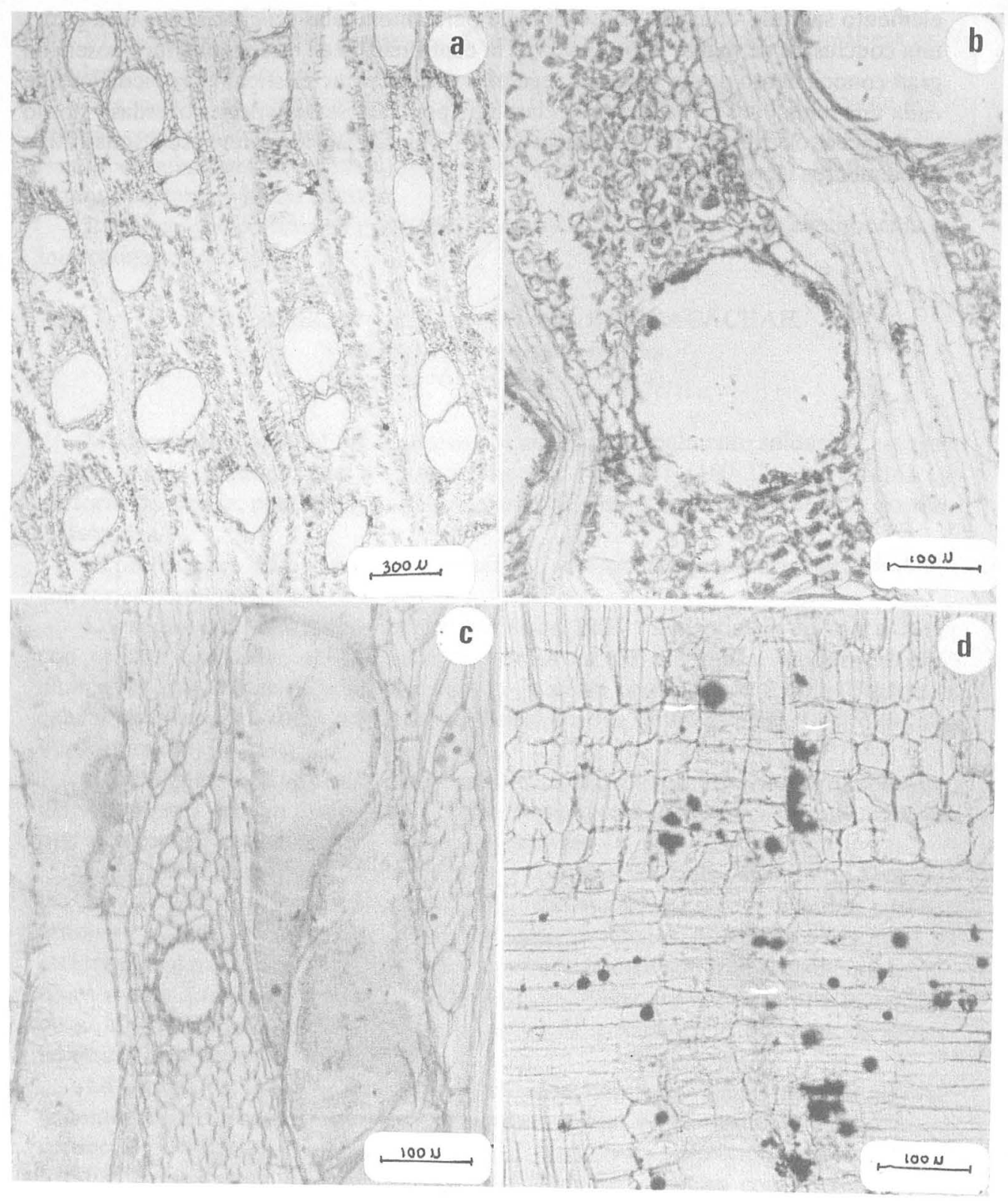

Fig. 3. Spondias mombin L., escultura No. 12 del sitio El Manatí. a) sección transversal, b) sección transversal, c) sección tangencial, d) sección radial. 

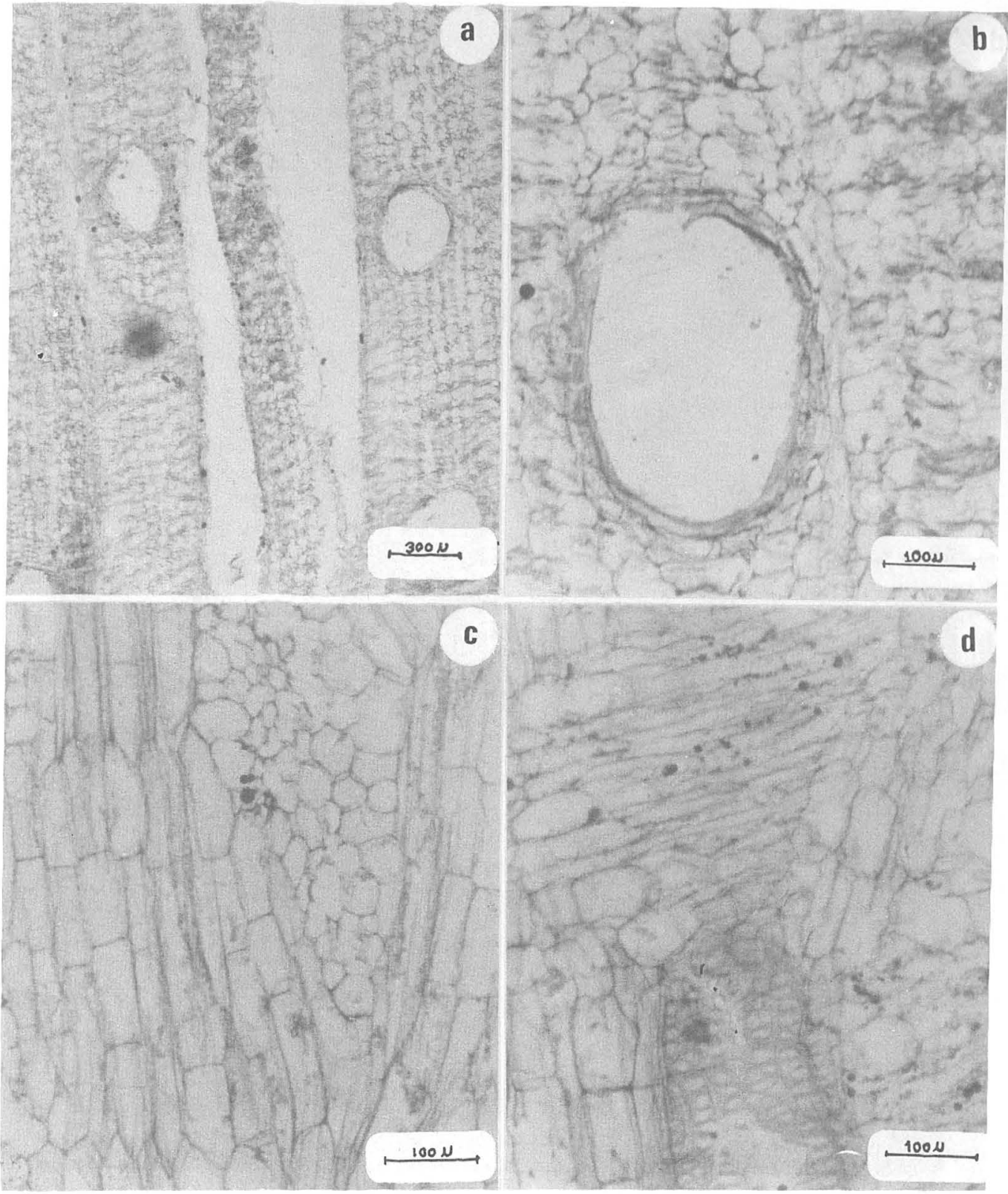

Fig. 4. Ceiba pentandra (L.) Gaertn escultura No. 2 del sitio El Manatí. a) sección transversal, b) sección tangencial, d) sección radial. 
BOLETIN DE LA SOCIEDAD BOTANICA DE MEXICO, Núm. 51, 1991

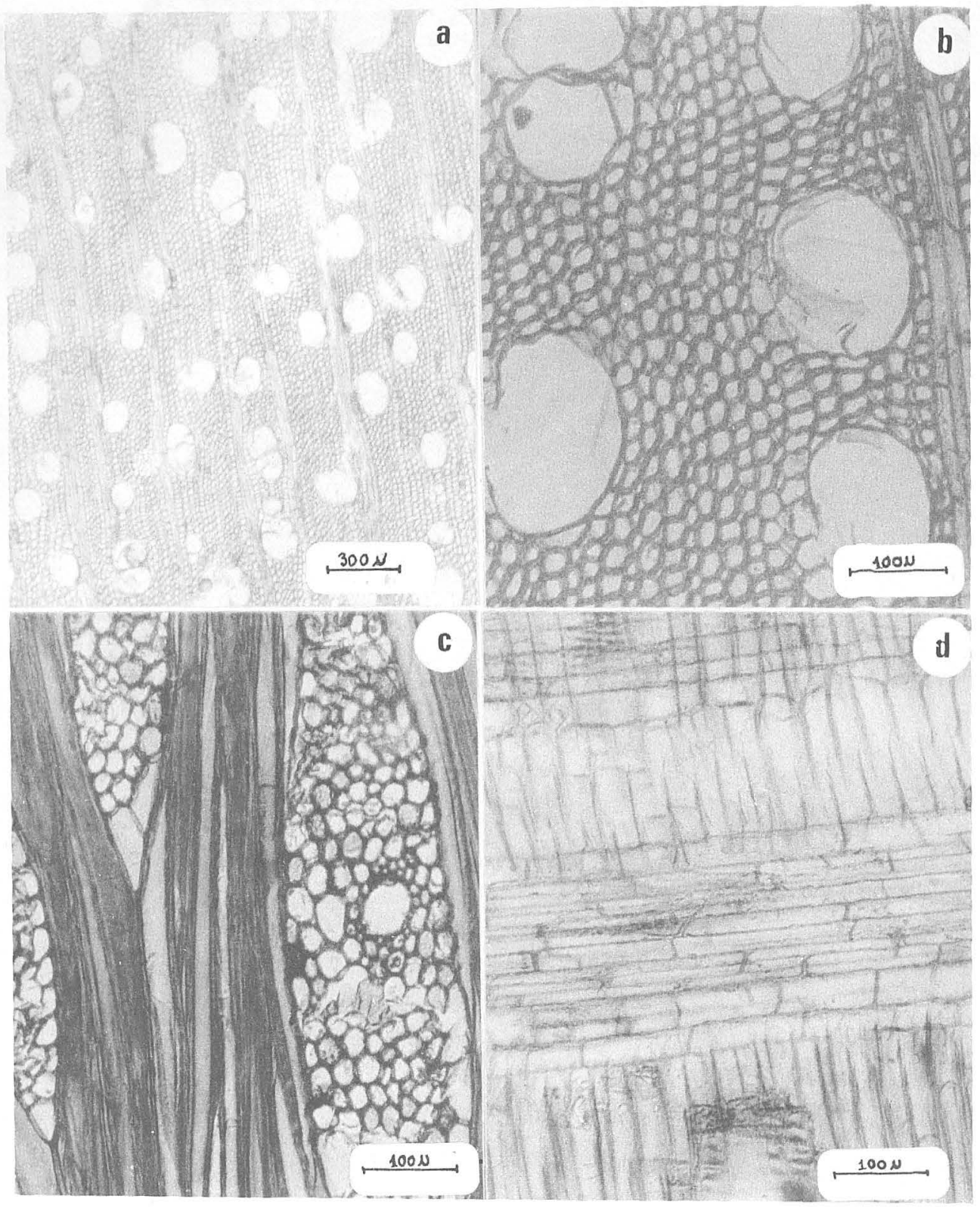

Fig. 5. Spondias mombin L., muestra MEXU: (M. Ortega), material actual a) sección transversal, b) sección transversal, c) sección tangencial, d) sección radial. 


\section{NOTAS BOTANICAS}

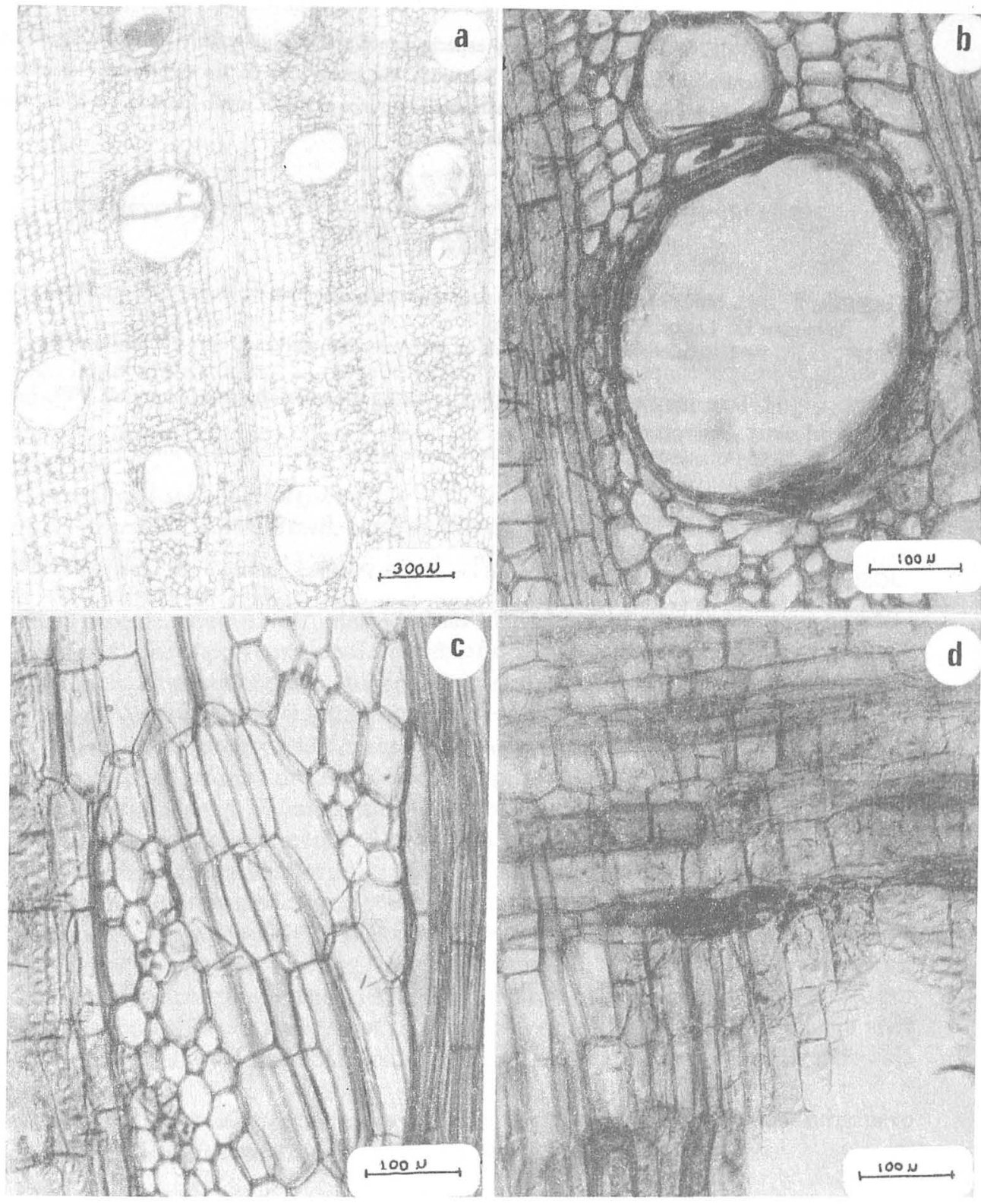

Fig. 6. Ceiba pentandra (L.) Gaertn muestra INIREB-053, material actual. a) sección transversal, b) sección transversal, c) sección tangencial, d) sección radial. 
AgRADECIMIENTOS. Agradezco muy especialmente al Dr. Guillermo Angeles su ayuda en la selección de la técnica utilizada, al Dr. Paul Schmidth, las fotografías de las esculturas y el mapa de localización del sitio El Manatí. De la misma manera se agradece al Biól. Calixto León G. y a la estudiante Susana López H. la realización del trabajo de laboratorio.

\section{LITERATURA CITADA}

GARCÍA, E. 1981. Modificaciones al sistema de clasificación climática de Koppen. 3a ed. Publicađo por la autora. Ed. Larios, México, D.F. 252p.

KRIBS, D.A. 1968. Commercial foreign woods on the American market. Dover Publications, New York. 241p.

ORTíz, P., H. RodríguEZ, y P. SCHMIDT. 1988. El proyecto Manatí. Temporada 1988. Informe preliminar. Arqueología 3:140-154.

SASS, J.E. 1958. Botanical microtechnique. 3a ed. Iowa State University Press. Ames. 228p.

JOSEFINA BARAJAS MORALES. Departamento de Botánica, Instituto de Biología, Universidad Nacional Autónoma de México, Apdo. Postal 70-233, Delegación Coyoacán, 04510, México, D.F. 